\title{
Spectrum and frequencies of mutations in the MFN2 gene and its phenotypical expression in Czech hereditary motor and sensory neuropathy type II patients
}

\author{
DANA ŠAFKA BROŽKOVÁ ${ }^{1}$, JAN POSÁDKA ${ }^{1}$, PETRA LAŠŠUTHOVÁ ${ }^{1}$, RADIM MAZANEC $^{2}$, \\ JANA HABERLOVÁ ${ }^{1}$, DANA ŠIŠKOVÁ ${ }^{3}$, IVA SAKMARYOVÁ ${ }^{1}$, JANA NEUPAUEROVÁ ${ }^{1}$ and PAVEL SEEMAN ${ }^{1}$ \\ ${ }^{1}$ DNA Laboratory, Department of Child Neurology; ${ }^{2}$ Department of Neurology, \\ Charles University Second Medical School and University Hospital Motol, Prague 15006; \\ ${ }^{3}$ Department of Child Neurology, Thomayer University Hospital, Prague 14059, Czech Republic
}

Received April 24, 2013; Accepted October 3, 2013

DOI: $10.3892 / \mathrm{mmr} .2013 .1730$

\begin{abstract}
The axonal type of Charcot-Marie-Tooth (CMT) disorders is genetically heterogeneous, therefore the causal mutation is unlikely to be observed, even in clinically well characterized patients. Mitofusin-2 (MFN2) gene mutations are the most frequent cause of axonal CMT disorders in a number of populations. There are two phenotypes; early onset, which is severe and late onset, which is a milder phenotype. A cohort of 139 unrelated Czech patients with axonal neuropathy was selected for sequencing and multiplex ligation-dependent probe amplification analysis (MLPA) testing of the MFN2 gene. A total of 11 MFN2 mutations were detected, with eight pathogenic mutations and three potentially rare benign polymorphisms. MLPA testing in 64 unrelated patients did not detect any exon duplication or deletion. The frequency of the pathogenic mutations detected in Czech hereditary motor and sensory neuropathy type II (HMSN II) patients was 7.2\%. Early onset was more frequent among pathogenic mutation cases. Therefore we propose to examine the $M F N 2$ gene mainly in patients with early and severe axonal CMT.
\end{abstract}

\section{Introduction}

Heterogeneous Charcot-Marie-Tooth (CMT) neuropathy is traditionally divided into a demyelinating or axonal type based on the peripheral nerve conduction velocity of $38 \mathrm{~m} / \mathrm{sec}$ on

Correspondence to: Dr Dana Šafka Brožková, DNA Laboratory, Department of Child Neurology, Charles University Second Medical School and University Hospital Motol, 84 V Úvalu, Prague 15006, Czech Republic

E-mail: dana.brozkova@seznam.cz

Key words: axonal neuropathy, Mitofusin-2 gene, Charcot-Marie-Tooth neuropathy, hereditary motor and sensory neuropathy the median nerve (1). A number of genes are implicated in the demyelinating type (type I) whereas, only a few are known for the axonal type (type II) $(2,3)$. To date, the most frequently reported mutated gene for axonal neuropathy is the mitofusin-2 (MFN2) gene, and it was first reported in 2004 (4). The MFN2 mutations were found in $\leq 20 \%$ of HMSN II patients. Phenotypes of axonal neuropathy due to a MFN2 mutation may be divided in two groups; severe, early onset, which is more frequent, usually associated with de novo mutations and patients usually lose their ability to walk independently prior to 20 years of age. The late onset type is less frequent and usually familial, associated with inherited dominant mutations.

The MFN2 gene encodes for mitochondrial membrane protein, which plays a role in mitochondrial fusion and fission (5). To date, 110 mutations in the MFN2 gene are known (6). The current study reports the results from diagnostic testing of 139 unrelated patients with axonal HMSN neuropathy tested for MFN2 mutations. The clinical phenotype of patients with mutations in the MFN2 gene is described and the character of the novel mutations in the MFN2 gene is discussed.

\section{Patients and methods}

A total of 139 Czech patients with sporadic or familial presence of axonal neuropathy compatible with HMSN II were selected for MFN2 testing. All examined patients signed informed consent for DNA testing to clarify the cause of the hereditary neuropathies and the study was approved by the Ethics Committee of University Hospital Motol (Prague, Czech Republic). The majority of the selected patients were previously tested negative for the most common cause of CMT-CMT1A duplication/hereditary neuropathy with liability to pressure palsies deletion using the set of 17 microsatellite markers (7), patients with late onset were previously tested negative for mutations in the $M P Z$ and patients with early onset HMSN II were previously tested negative for mutations in the GDAPl gene.

All 17 coding exons and adjacent intron sequences were amplified in 12 PCR fragments with a set of primers (avail- 
able upon request) and annealing temperature of $65^{\circ} \mathrm{C}$. The sequencing reaction was performed for 15 fragments using BigDye Terminator v3.1 sequencing kit and products were analyzed on an ABI 3130 Genetic Analyzer (all Applied Biosystems, Inc., Foster City, CA, USA). The reference sequence used for MFN2 was NM_014874.3. When the presence of a mutation was observed, samples from additional family members were analyzed to evaluate the segregation of the mutation with the disease.

When the mutation was observed, the prediction programs SIFT (8) and PolyPhen-2 (9) were used for the pathogenicity classification of the mutations. Exome Variant Server (EVS) (10), 1000 Genomes $(11,12)$ and Single Nucleotide Polymorphisms database (dbSNP) $(13,14)$ were used to find information on the variants. The EVS operates with more than 6,500 samples analyzed for the exome variants, the current group was assumed to be large enough and therefore the 100 DNA control samples were not analyzed as was usual previously.

In addition, 73 patients from 64 families, previously tested negative for the MFN2 mutations, were examined with the multiplex ligation-dependent probe amplification analysis method (MRC Holland, Amsterdam, The Netherlands) to exclude larger rearrangements (duplications, deletions) not detectable with Sanger sequencing. The SALSA MLPA kit, P143-MFN2-MPZ was used and analysis was performed according to the manufacturer's instructions. Products were analyzed on an ABI 310 Genetic Analyzer. GeneMapper software v4.0 and Coffalyser v1.0.0.43 (MRC Holland) were used to analyze the data.

\section{Results}

A total of 11 nonsynonymous heterozygous mutations were detected by sequencing in 15 unrelated patients from a group of 139 patients with axonal neuropathy (Fig. 1). A total of eight were pathogenic, the remaining three were observed in healthy relatives and are therefore considered as rare benign polymorphisms (Table I). The frequency of detected pathogenic MFN2 mutations in the cohort was $7.2 \%$. The prediction programs support the present observations, with the exception of two cases; p.L209Q was classified as pathogenic, but was also observed in a healthy relative and p.W740S was scored by PolyPhen-2 as benign but was already reported as disease causing $(4,15)$. Five from the 11 reported mutations are novel. No exonic deletion or duplication of any of the 17 exons examined was detected with MLPA in the 73 patients from 64 families. A large number of previously reported polymorphisms was observed. An overview of all observed polymorphisms with minor allele frequency is summarized in Table II.

Families with pathogenic mutations. A novel pathogenic mutation, p.E65X, was observed in a mother and her daughter. The mother was examined at 58 years of age; the patient was capable of walking without support and only mild foot deformity and arched instep were present. The daughter exhibited the first signs of CMT in childhood and subsequently underwent three orthopedic corrective surgeries on the both feet. At the age of 29 , the patient is capable of walking without support, but has muscle atrophies below the knees and on the upper limbs.

The mutation p.R94W, was observed in two patients from different families. Disease onset was early, 4-5 years of age in the patients and the two were severely affected. The two patients were $\sim 30$ years of age and suffer from pronounced muscle atrophies extending above the knees and severe atrophies of the small hand muscles were present (Fig. 2). Both patients had gait problems and required crutches. Muscle strength was reduced in the wrist to $2 / 2$ (MRC) in patient $2 \mathrm{~A}$ and $3 / 3$ in patient $2 \mathrm{~B}$. Muscle strength in the ankles was reduced to 0 in both patients.

Mutation p.T105R was observed in two sisters affected by axonal CMT neuropathy. The older sibling was more severely affected, with the affliction beginning at 4 years of age. From the age of 10 years, the patient had been using a wheelchair. Following surgical prolongation of the Achilles tendons, the patient is capable of walking with the support of canes. At the current age of 28 years, the patient exhibits severe atrophies above the knees and marked scoliosis. In her younger sister, the disease manifested at the age of 14 years. At the current age of 23 years, the patient exhibits mild atrophies distally in the legs and pes cavus deformities. The two sisters are heterozygotes for the same mutation and it was not observed in their parents. Examination using 17 microsatellite markers proved correct parenthood for the parents, thus, a gonadal mosaicism in one of the parents is the most probable explanation.

The known mutation p.H165Y was detected in two families with similar phenotypes. In the two families, the disease manifested early, 6-10 years of age. The father (47 years) and daughter (26 years) from the first family were severely affected. They underwent a number of orthopedic corrective surgeries on their feet and are capable of walking with the support of canes. The patients have minor atrophies of the small hand muscles. The father (54 years of age) and two sons (27 and 29 years of age) are affected in the second family (Fig. 2). The father and older son had a number of orthopedic corrective surgeries on their feet. The older brother is more severely affected compared with the father and has atrophies of the small hand muscles. The affected males from the second family are capable of walking without support.

Mutation p.A166T was observed in a mother and son who are mildly affected. The mother has had difficulties with walking since childhood and the last examination at the age of 51 years revealed distal atrophies of the leg muscles, foot deformities and shortened Achilles tendons. The son was examined at the age of 34 years and clinically exhibited no manifestations of CMT neuropathy. Axonal neuropathy was observed upon electrophysiological examination.

Mutation p.R707W was observed in a 14-years old patient with axonal neuropathy in whom symptoms of CMT manifested $\sim 7$ years of age. The patient exhibited muscle atrophies of the lower limbs and atrophies of the small hand muscles. The patient's mother feels healthy and is declared as unaffected, but has the same mutation. However, the mother denied any clinical or electrophysiological examination.

Mutation p.W740S was observed in an affected patient and his daughter. The patient's sister, mother and uncle, had similar difficulties but did not agree to genetic testing. The patient has muscle atrophies of the lower limbs and foot deformities. The 


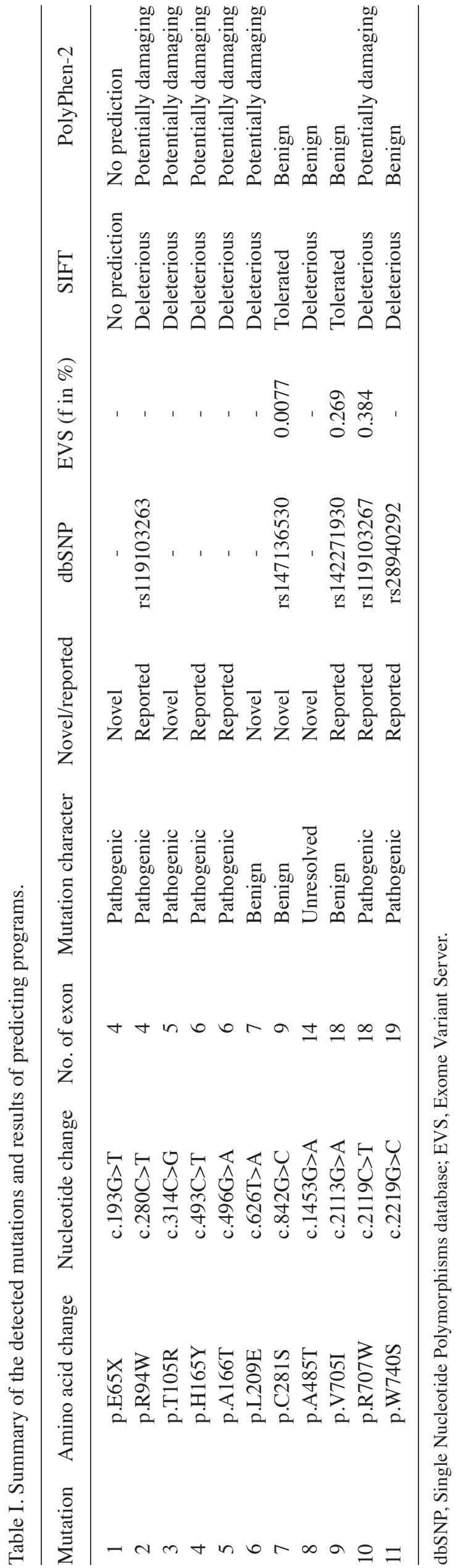

daughter was clinically examined at 5 years of age. The patient stumbles and has flat feet. Electrophysiological examination was refused by the parents.

Family with a probable pathogenic mutation. Mutation p.A485T was observed in a single patient with axonal neuropathy which manifested at the age of 55 years. Electrophysiological examination confirmed the axonal neuropathy. The patient is currently 70 years old and has no children for testing of segregation in the family.

Families with probable benign variants. Mutations p.L209E and p.C281S were observed in three families with axonal HMSN. The same mutations were also observed in their healthy relatives, but clinical and electrophysiological examination revealed no signs of CMT neuropathy. Mutation p.V705I was observed in two patients from two unrelated families. The 70 years old patient in the first family has presented with problems since childhood and his clinical phenotype is similar to CMT1A, but exhibits axonal neuropathy according to electrophysiological examination. A patient from the second family has had problems from 33 years of age. The patient has complicated diabetes mellitus and after the neurological and electrophysiological examination the HMSN is suspected.

Within this cohort with pathogenic mutations two clusters of phenotypes were observed. i) Severe phenotype with age of onset occurring at 4-6 years but with normal early motor development and walking beginning at $\sim 12$ months of age was observed more frequently ( 6 of 10 pathogenic mutations, $60 \%$ ). Patients with this phenotype usually lost their ability of independent walking prior to the age of 20 years and require crutches or a wheelchair. These patients are usually sporadic cases in the families and are a result of a mutation which arose de novo. The mutations with the de novo origin causing a severe phenotype are p.R94W and p.T105R. The familial cases are p.H165Y and p.W740S. ii) The second phenotype is mild with the age of onset between the 2nd and 5 th decade without loss of independent walking. This phenotype is usually in families with more affected members. This phenotype was observed in patients with p.E65X, p.A166T and p.R707W mutations.

\section{Discussion}

Pathogenic mutations were detected in 7.2\% (139/10) of unrelated Czech Charcot-Marie-Tooth neuropathy type 2 (CMT2) patients. Compared with Casasnovas et al (16) and Feely et al (17) who reported 16\% for the Spanish population and $21 \%$ for US and UK patients, the lower percentage in the current study may be due to less stringent selection criteria that was used and inclusion of more patients with late onset axonal neuropathy and also due our to participation in a previous collaborative study in 2006 (15), where unresolved severely affected HMSN II patients were selected and in 6 patients of 42 , the pathogenic mutation was detected.

A number of mutations detected in the current patient cohort in this study have previously been reported, namely; p.R94W, p.H165Y, p.A166T, p.V705I, p.R707W and p.W740S. Two patients with the p.R94W mutation are early and severely affected with HMSN II but no other clinical signs, including 
Table II. Summary of detected polymorphisms with known MAF and rs numbers.

\begin{tabular}{|c|c|c|c|}
\hline Position & Amino acid change & MAF & dbSNP \\
\hline c. $176-76 \mathrm{G}>\mathrm{A}$ & - & NA & NA \\
\hline c. $474+65 \mathrm{C}>\mathrm{T}$ & - & $C=0.366$ & rs2236056 \\
\hline c. $600-25 \mathrm{~T}>\mathrm{C}$ & - & $\mathrm{C}=0.058$ & rs41278626 \\
\hline c. $709-32 \mathrm{C}>\mathrm{T}$ & - & $\mathrm{T}=0.0009$ & rs41278628 \\
\hline c. $870 \mathrm{C}>\mathrm{T}$ & p.Gly290Gly & NA & NA \\
\hline c. $.957 \mathrm{C}>\mathrm{T}$ & p.Gly319Gly & $\mathrm{TT}=0.004$ & rs41278632 \\
\hline c.1039-22T>C & - & $\mathrm{C}=0.044$ & rs6680984 \\
\hline c.1496-34T>C & - & $\mathrm{C}=0.008$ & rs143065633 \\
\hline c. $1641 \mathrm{C}>\mathrm{T}$ & p.Leu547Leu & NA & rs140924661 \\
\hline c. $1872+63 \mathrm{~T}>\mathrm{C}$ & - & $\mathrm{C}=0.047$ & rs2273295 \\
\hline c. $1873-66 \mathrm{~T}-\mathrm{G}$ & - & $\mathrm{T}=0.469$ & rs7550536 \\
\hline c. $2204+15 \mathrm{~T}>\mathrm{C}$ & - & $\mathrm{C}=0.083$ & rs77262016 \\
\hline c. $* 187 \mathrm{C}>\mathrm{G}$ & - & NA & NA \\
\hline c. $* 231 \mathrm{G}>\mathrm{A}$ & - & $\mathrm{A}=0.011$ & rs41278636 \\
\hline c. $* 58 \mathrm{~A}>\mathrm{G}$ & - & $A=0.305$ & rs1042842 \\
\hline
\end{tabular}

dbSNP, Single Nucleotide Polymorphisms database; NA, not available; MAF, minor allele frequencies.

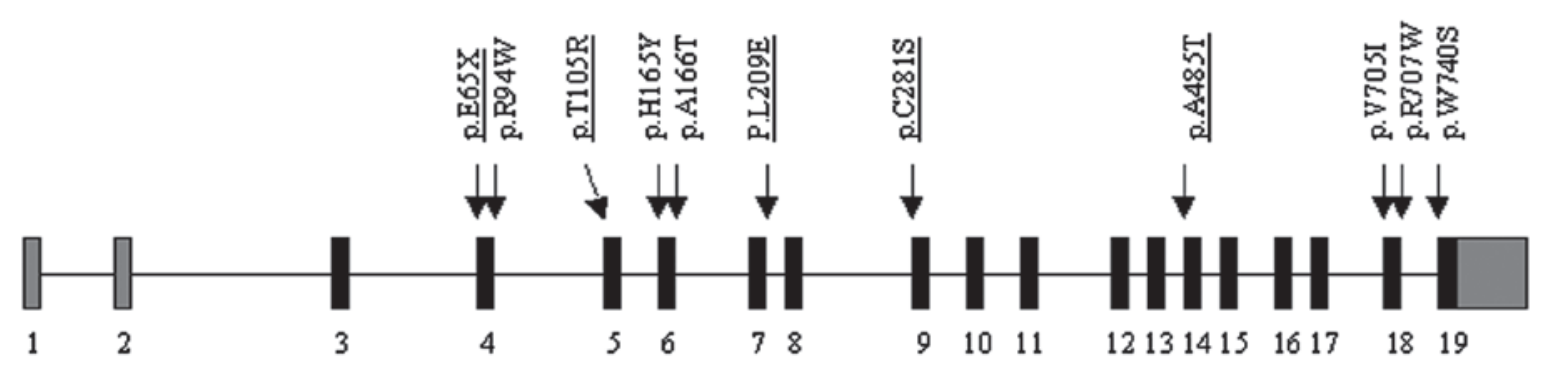

Figure 1. Mutations in the MFN2 gene detected in this study and their positions in exons. The MFN2 gene contains 17 coding and 2 non-coding exons. Novel mutations are underlined. MFN2, mitofusion-2.

optic atrophy or increased tendon reflexes were observed. The same was reported for this mutation in a number of other studies (15-20). Only one family with p.R94W mutation developed optic atrophy at ages 40 and 50 years (21).

The previously reported p.H165Y mutation was observed in a Czech family (15). It is highly probable that this family is related to the family reported in the present study since they have the same family name, however, it was not possible to prove this. Three other amino acid changes were reported for the same codon p.H165 in HMSN II patients; p.H165D in one family $(22)$, p.H165R in two single patients $(15,19)$ and one family with additional signs of tremor and sensorineural hearing loss (18) and p.H165L in a patient with mild to moderate predominantly motor CMT neuropathy up to the age of 60 years, followed by progression acceleration, proximal limb muscle weakness and bulbar involvement (23). The four substitutions showed that this codon, p.H165, is a mutation hotspot in the MFN2 gene, similar to p.R94 and others.

The p.A166T mutation was previously reported in a family where the mother is wheelchair-bound and unable to write at the age of 49 years. Her daughter exhibited distal muscle atrophies in all four limbs at the age of 28 years (24). The clinical findings are similar to the current observations where a 51-year-old mother is more severely affected compared with her son at age 34 years where CMT neuropathy was observed only upon electromyography examination.

The p.V705I mutation was previously reported as causing disease in one patient with CMT2 (25). This mutation was observed in two unrelated CMT2 patients. However, the SIFT and PolyPhen-2 scored it as benign and the frequency for mutated allele in EVS is $0.3 \%$. The mutation is therefore potentially a polymorphism and is not causal for CMT2 neuropathy in these patients or it may be a recessive mutation.

The mild phenotype in patients with the p.W740S mutation is similar to the phenotype previously reported for this mutation $(4,15,17)$.

In the MFN2 gene eight recessive mutations have been reported. The first compound heterozygous mutation was reported by Verhoeven et al (15), but without data from their parents. Nicholson et al (26) reported two compound heterozygotes and homozygous p.R707W in one patient where all parents were asymptomatic carriers of heterozygous 

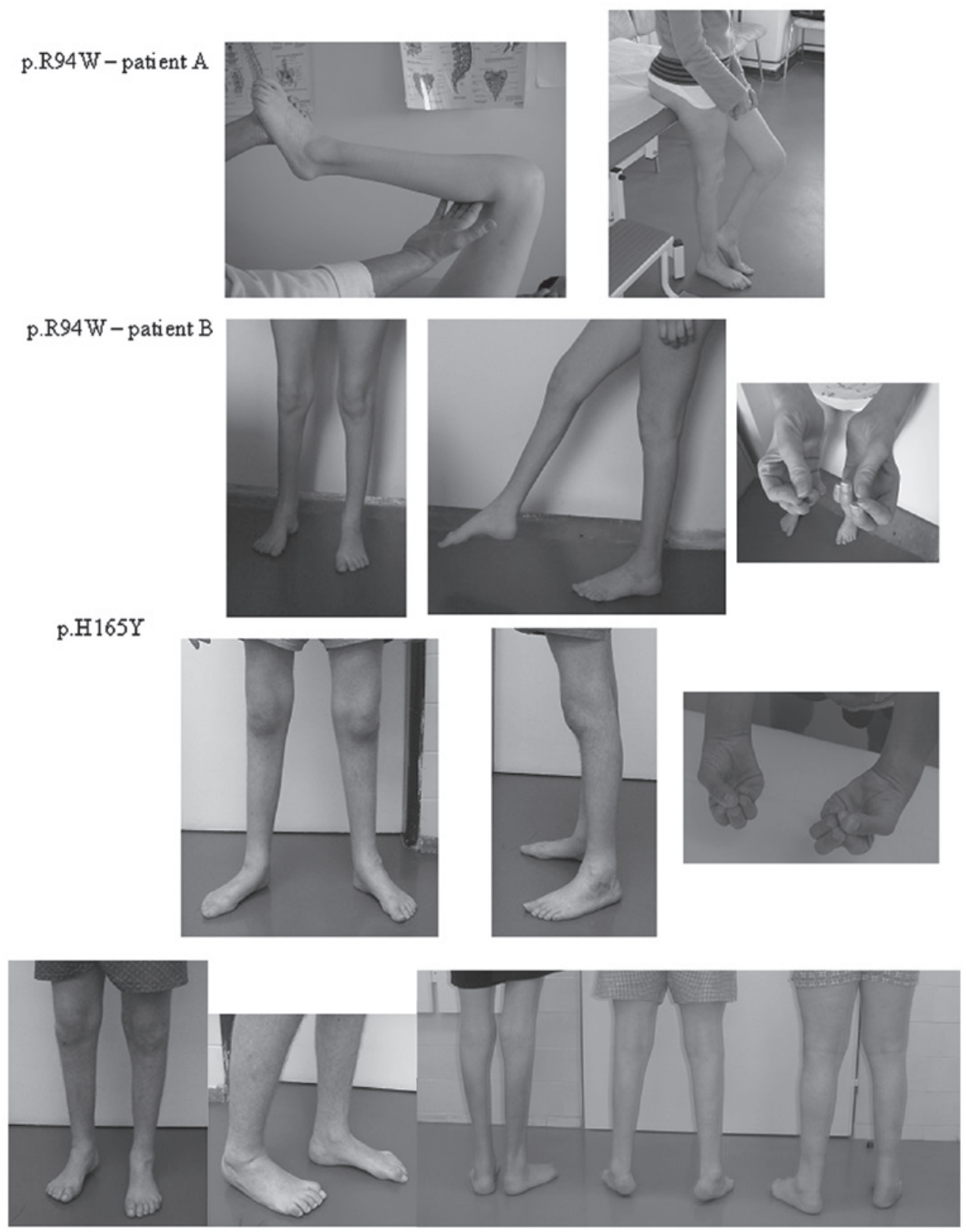

Figure 2. Images of patients with mutations p.R94W from family A and B and images of family with mutation p.H165Y (affected father and two sons).

mutations with minimal clinical findings of neuropathy. Other compound heterozygous mutations, p.G108R and p.R707W, were reported in three siblings by Calvo et al (20) where the parents had no signs of peripheral neuropathy, had normal electrophysiological findings and each carried a single heterozygous mutation. Polke et al (27) reported three patients with recessive mutations. In two siblings the combination of missense mutations and deletion of 2 exons was observed. A heterozygous p.R707W mutation was detected in one patient with peripheral neuropathy and also in her asymptomatic mother in this study. The second mutation was not detected by sequencing, however it is possible that it may be missed in a specific deep intronic part, thus, the causal effect of recessive p.R707W mutation remains possible.

Two of the five novel mutations are pathogenic. For p.G65X no prediction was possible, however, it is a stop mutation. p.G65X segregates in the family with the HMSN II phenotype and this variant is not present in EVS. The second pathogenic mutation, p.T105R, was detected in two sisters but not in their healthy parents with confirmed parentity, thus a gonadal mosaicism in one of the parents appears to be the most probable explanation. This mutation is not observed in EVS but another amino acid change, p.T105M, in the same codon was reported as causal and supports the current conclusion $(17,18,28)$. Two other mutations, p.L209E and p.C281S, are assumed to be benign variants or recessive mutations since they were also identified in healthy relatives with normal findings under electrophysiological examination.

The remaining novel mutation, p.A485T, was observed in a single patient, thus, the pathogenicity may not be clearly resolved. Moreover, the prediction by SIFT and PolyPhen-2 is tolerated and benign and the variant was not detected in EVS. For a more reliable prediction, another family with segregation of the mutation together with the phenotype is required. 
During examination of the Czech HMSN II patients for MFN2 mutations 11 heterozygous mutations were observed, five of them novel and six previously reported. The authors propose to examine the MFN2 gene primarily in patients with early and severe axonal CMT, where early motor development was normal, but where patients have pronounced distal weakness in preschool age and in patients with later onset of CMT2 between the 2 nd and 5 th decade with autosomal dominant inheritance in the family. MLPA examination did not reveal any duplication or deletion in MFN2 exons and these aberrations are not a relevant cause of CMT. Performing MPLA examination of the MFN2 gene in routine MFN2 gene testing is therefore neither effective nor rational.

\section{Acknowledgements}

The study was supported by grant no. IGA MH CZ NS 10554-3 and by MH CZ - DRO, University Hospital Motol, Prague,Czech Republic 00064203.

\section{References}

1. Harding AE and Thomas PK: The clinical features of hereditary motor and sensory neuropathy types I and II. Brain 103: 259-280, 1980.

2. Inherited Peripheral Neuropathies Mutation Database. http://www.molgen.ua.ac.be/CMTMutations/Home/IPN.cfm. Accessed October 3, 2013.

3. Züchner S and Vance JM: Molecular genetics of autosomal-dominant axonal Charcot-Marie-Tooth disease. Neuromolecular Med 8: 63-74, 2006.

4. Züchner S, Mersiyanova IV, Muglia M, et al: Mutations in the mitochondrial GTPase mitofusin 2 cause Charcot-Marie-Tooth neuropathy type 2A. Nat Genet 36: 449-451, 2004.

5. Nunnari J, Marshall WF, Straight A, Murray A, Sedat JW and Walter P: Mitochondrial transmission during mating in Saccharomyces cerevisiae is determined by mitochondrial fusion and fission and the intramitochondrial segregation of mitochondrial DNA. Mol Biol Cell 8: 1233-1242, 1997.

6. Stenson PD, Mort M, Ball EV, et al: The Human Gene Mutation Database: 2008 update. Genome Med 1: 13, 2009.

7. Seeman P, Mazanec R, Zidar J, Hrusáková S, Ctvrtecková M and Rautenstrauss B: Charcot-Marie-Tooth disease type 1A (CMT1A) and hereditary neuropathy with liability to pressure palsies (HNPP): reliable detection of the CMT1A duplication and HNPP deletion using 8 microsatellite markers in 2 multiplex PCRs. Int J Mol Med 6: 421-426, 2000.

8. Ng PC and Henikoff S: Predicting deleterious amino acid substitutions. Genome Res 11: 863-874, 2001.

9. Sunyaev S, Ramensky V, Koch I, Lathe W 3rd, Kondrashov AS and Bork P: Prediction of deleterious human alleles. Hum Mol Genet 10: 591-597, 2001.
10. NHLBI Exome Sequencing Project (ESP): Exome Variant Server (EVS). http://evs.gs.washington.edu/EVS. Accessed October 3, 2013.

11. 1000 Genomes Project Consortium; Abecasis GR, Altshuler D, Auton A, et al: A map of human genome variation from population-scale sequencing. Nature 467: 1061-1073, 2010.

12. 1000 Genomes. http://www.1000genomes.org. Accessed October 3, 2013.

13. Sherry ST, Ward MH, Kholodov M, et al: dbSNP: the NCBI database of genetic variation. Nucleic Acids Res 29: 308-311, 2001.

14. National Center for Biotechnology Information, National Library of Medicine: Database of Single Nucleotide Polymorphisms (dbSNP). http://www.ncbi.nlm.nih.gov/SNP. Accessed October 3, 2013.

15. Verhoeven K, Claeys KG, Zuchner S, et al: MFN2 mutation distribution and genotype/phenotype correlation in Charcot-Marie-Tooth type 2. Brain 129: 2093-2102, 2006.

16. Casasnovas C, Banchs I, Cassereau J, et al: Phenotypic spectrum of MFN2 mutations in the Spanish population. J Med Genet 47: 249-256, 2010.

17. Feely SM, Laura M, Siskind CE, et al: MFN2 mutations cause severe phenotypes in most patients with CMT2A. Neurology 76: 1690-1696, 2011

18. Chung KW, Kim SB, Park KD, et al: Early onset severe and late-onset mild Charcot-Marie-Tooth disease with mitofusin 2 (MFN2) mutations. Brain 129: 2103-2118, 2006.

19. Cho HJ, Sung DH, Kim BJ and Ki CS: Mitochondrial GTPase mitofusin 2 mutations in Korean patients with Charcot-Marie-Tooth neuropathy type 2. Clin Genet 71: 267-272, 2007.

20. Calvo J, Funalot B, Ouvrier RA, et al: Genotype-phenotype correlations in Charcot-Marie-Tooth disease type 2 caused by mitofusin 2 mutations. Arch Neurol 66: 1511-1516, 2009.

21. Züchner S, De Jonghe P, Jordanova A, et al: Axonal neuropathy with optic atrophy is caused by mutations in mitofusin 2 . Ann Neurol 59: 276-281, 2006.

22. Zhu D, Kennerson ML, Walizada G, Züchner S, Vance JM and Nicholson GA: Charcot-Marie-Tooth with pyramidal signs is genetically heterogeneous: families with and without MFN2 mutations. Neurology 65: 496-497, 2005.

23. Marchesi C, Ciano C, Salsano E, et al: Co-occurrence of amyotrophic lateral sclerosis and Charcot-Marie-Tooth disease type $2 \mathrm{~A}$ in a patient with a novel mutation in the mitofusin-2 gene. Neuromuscul Disord 21: 129-131, 2011.

24. Loiseau D, Chevrollier A, Verny C, et al: Mitochondrial coupling defect in Charcot-Marie-Tooth type 2A disease. Ann Neurol 61: 315-323, 2007.

25. Engelfried K, Vorgerd M,Hagedorn M,et al: Charcot-Marie-Tooth neuropathy type 2A: novel mutations in the mitofusin 2 gene (MFN2). BMC Med Genet 7: 53, 2006.

26. Nicholson GA, Magdelaine C, Zhu D, et al: Severe early-onset axonal neuropathy with homozygous and compound heterozygous MFN2 mutations. Neurology 70: 1678-1681, 2008.

27. Polke JM, Laurá M, Pareyson D, et al: Recessive axonal Charcot-Marie-Tooth disease due to compound heterozygous mitofusin 2 mutations. Neurology 77: 168-173, 2011.

28. Lawson VH, Graham BV and Flanigan KM: Clinical and electrophysiologic features of CMT2A with mutations in the mitofusin 2 gene. Neurology 65: 197-204, 2005. 